\title{
Modeling and analysis of IEEE 1609.4 MAC in the presence of error-prone channels
}

\author{
Akram A. Almohammedi ${ }^{1}$, Nor K. Noordin², A. Sali ${ }^{3}$, Fazirulhisyam Hashim ${ }^{4}$, \\ Waheb A. Jabbar ${ }^{5}$, Sabri Saeed ${ }^{6}$ \\ ${ }^{1,2,3,4}$ Department of Computer and Communication Engineering, Universiti Putra Malaysia, Malaysia \\ ${ }^{2,3,4}$ Wireless and Photonics Networks Laboratoratory, Universiti Putra Malaysia, Malaysia \\ ${ }^{5}$ Faculty of Engineering Technology, Universiti Malaysia Pahang, Malaysia \\ ${ }^{6}$ Department of Communication Systems Engineering, Taiz University, Taiz-Yemen
}

\begin{tabular}{l} 
Article Info \\
\hline Article history: \\
Received Jul 19, 2018 \\
Revised Apr 4, 2019 \\
Accepted Apr 15, 2019 \\
\hline Keywords: \\
Back-off freezing timer \\
Error-prone channels \\
IEEE 1609.4 \\
Performance analysis \\
VANETs
\end{tabular}

\begin{abstract}
Vehicular Ad Hoc Networks (VANETs) have been developed to improve the safety, comfort and efficiency of driving on the road. The IEEE 1609.4 is a standard intended to support multi-channel in VANETs. These channels include one control channel for safety applications and six service channels for service applications. However, there is still no comprehensive analysis for the average delay and system throughput of IEEE 1609.4 MAC in VANETs considering error-prone channel under non-saturated conditions. In this paper, we propose an analytical models based on 1-D and 2-D Markov chain to evaluate the performance analysis of IEEE 1609.4-based MAC in the presence of error-prone channels. Besides, freezing of the back-off timer is taken into consideration to provide an accurate estimation of access to the channel. The simulation results have been carried out to validate the analytical results of our model. The results show that the performance of our model outperforms the existing model in terms of packet delivery ratio and average delay of safety packets over $\mathrm{CCH}$, and system throughput of service packets over SCHs.
\end{abstract}

Copyright $@ 2019$ Institute of Advanced Engineering and Science. All rights reserved.

\section{Corresponding Author:}

Akram A. Almohammedi

Department of Computer and Communication Systems Engineering,

Universiti Putra Malaysia,

43300 Serdang, Malaysia.

Email: akrama2810@gmail.com

\section{INTRODUCTION}

Recently, with increasing the population, the number of registered vehicles has dramatically increased over all the world, and this leads to a high rate of traffic accidents on the roads. In order to prevent such accidents, an Intelligent Transportation Systems (ITSs) are needed. ITSs typically has the ability to improve the quality, effectiveness and safety of the future transportation systems. However, VANETs are the key component of ITSs which integrate wireless networks into vehicles. VANETs support three types of communications including Vehicle-to-Vehicle (V2V), Vehicle-to-Infrastructure (V2I), and Hybrid Vehicular (HV) communications. The applications of VANETs are divided into two categories, safety applications and service applications. Safety applications are used to notify drivers about the critical situation in advance. On the other hand, service applications are used for improving driving comfort and the efficiency of transportation. As a result, safety applications are delay-sensitive and have a higher priority, while service applications are throughput-sensitive and have lower priority. The US Federal Communications Commission (FCC) has allocated a frequency band of $5.9 \mathrm{GHz}$ in a total bandwidth of $75 \mathrm{MHz}$ under DSRC to support 7-channels each of which is $10 \mathrm{MHz}$ wide and the guard band is $5 \mathrm{MHz}$ wide. These channels are functionally divided into one control channel ( $\left.\mathrm{CCH}_{-} 178\right)$, and up to six are service channels (SCHs) [1-3]. 
The $\mathrm{CCH}$ is exclusively used to broadcast safety-critical applications and regular traffics, while the six other channels, SCHs, are dedicated to transfer service data applications. Wireless Access in Vehicular Environment (WAVE) has been designed for VANETs based on the IEEE 802.11p and IEEE 1609.x standards family. The IEEE 802.11p standard supports both the physical (PHY) and medium access control (MAC) layers of DSRC. The IEEE 1609.4 is the standard (legacy) intended to support multi-channel operation in VANETs [1-3]. The repeating synchronization intervals (SI) among the channels to transmit the packets are $100 \mathrm{~ms}$, and each SI is evenly divided into $50 \mathrm{~ms}$ for $\mathrm{CCH}$ Interval (CCHI) and $50 \mathrm{~ms} \mathrm{SCH}$ Interval (SCHI) as shown in Figure 1 [1-5].

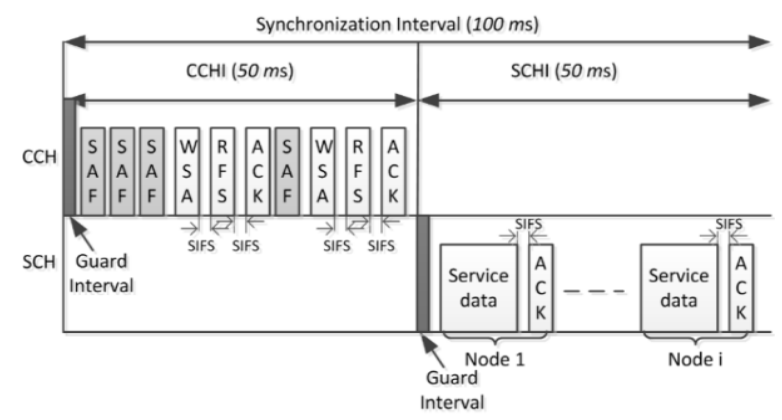

Figure 1. Multi-channel MAC operation in VANETs-IEEE 1609.4

For SCHs reservation in IEEE 1609.4, vehicle (provider) initially broadcasts WAVE Service Advertisement (WSA) packet on the $\mathrm{CCH}$ during $\mathrm{CCHI}$ to advertise its service and select an appropriate $\mathrm{SCH}$ for this service. If other vehicles (users) interest to join the service, which is advertised by a provider, will reply by Request for Service (RFS). Then, the provider replies the users by Acknowledgment (ACK) either for acceptance or rejection. Typically, packets collision may occur if more than one vehicle starts transmitting a packet simultaneously within the same time slot. Whereas, transmission error may manifest due to the complex condition of a wireless channel in VANETs such as path loss, thermal noise, channel fading, or interference from other radio resources.

The principle analysis of IEEE 802.11 Distributed Coordination Function (DCF) was introduced by Bianchi [6]. Bianchi proposed 2-D Markov Chain model to analyze the performance of MAC DCF mechanism by computing the throughput, assuming saturated traffic and error free channel. Several works such as [7-9] followed Bianchi's model by analyzing the throughput and delay of IEEE 802.11 DCF under saturated traffic taking into consideration frame retry limits, error-prone channels and freezing of the backoff timer. Unlike saturated traffic, the analytical model for the throughput and delay of IEEE 802.11 DCF performance under non-saturated traffic was studied by [10-11]. For the broadcast analysis in VANETs, several works such as [12-14] have evaluated the saturation performance of safety messages broadcast in VANETs to calculate the throughput and delay for emergency and routine messages. Different from broadcast analysis in VANETs, the unicast performance analysis of IEEE $802.11 \mathrm{p}$ in the presence of hidden terminals under both saturated and unsaturated traffics was presented in [15]. The analytical model of VANETs including both broadcast and unicast analysis for safety and service applications, respectively, based on Markov chain model were introduced in [16]. The authors in [16] analyzed the performance of IEEE $802.11 \mathrm{p}$ based MAC under non-saturated traffic and error free channels. The performance metrics of delay, packet delivery ratio and system throughput were investigated in [16]. The authors in [17-18] offered the analytical study of the IEEE 1609.4 MAC in VANETs under non-saturated condition. The performance metrics of delay, packet delivery ratio and system throughput were studied in [17-18]. However, freezing of the back-off timer and error-prone channels were not taken into consideration in [17].

Our model is extension of the existing model in [17]. Freezing of the back-off timer with the $M / M / 1$ queue and error-prone channels are taken into considered in our model. Taking these elements into account will provide an accurate estimation of access to the channel and also avoid the overestimation of the system throughput. However, this paper focuses on analyzing two types of traffics; safety and service traffics with higher and lower priorities respectively. 1-D and 2-D Markov chain are employed to model the back-off procedures for each traffic type in the presence of error-prone channels under non-saturated conditions. Gaussian wireless error channel is adopted in this model, in which a constant channel bit error rate (BER) is supposed to be identified in advance and each bit has the same bit error probability. The performance metric PDR, average delay of safety applications and system throughput of service applications are investigated in this paper to evaluate the performance analysis of the IEEE 1609.4. 


\section{ANALYTICAL MODEL}

\subsection{Multi-priority transmission model}

This analytical model considers the IEEE 1609.4 standard specifications for the safety and service applications as displayed in Figure 1. There are $n$ vehicles in the network contending to access the channel based on the EDCA scheme. All vehicles are in the transmission range of each other and there are no hidden terminals in the system. However, according to the IEEE 1609.4 standard, safety and WSAs packets are only transmitted over $\mathrm{CCH}$ during $\mathrm{CCHI}$ as shown in Figure 1. If safety or WSAs packets arrive at MAC layer during the SCHI at rate $\lambda$, they have to queue at MAC layer buffer waiting for the subsequent CCHI to be transmitted. Thus, under heavy traffic, many packets will be queued at MAC layer buffer waiting for transmission at the beginning of the next CCHI. This will increase collision and delay of transmitted safety packets, and decrease the PDR and accordingly the performance of VANETs will be degraded. The critical solution to achieve a reliable dissemination of safety packet on the road is to mitigate the conflict when accessing the $\mathrm{CCH}$. Thus, in order to mitigate the collision probability over $\mathrm{CCH}$, the considered application layer has to schedule the generated packets to arrive at MAC layer with Poisson manner by delaying a time of SCHI $(50 \mathrm{~ms})$. Meaning that there will be two queues with the same arrival rate $\lambda$ during the CCHI. The total of two independent Poisson process with rate $\lambda$ is $2 \lambda$. Thereby, the packet arrival rate for safety and WSAs traffic during the CCHI are denoted by $\lambda_{e}$ and $\lambda_{s}$, respectively, and they follow the Poisson distribution.

Let $b_{e}(t)$ be the random process representing the back-off timer value $\left(0,1,2, \ldots, W_{e}-1\right)$ at time slot $t$, while The back-off state process is denoted by $(k) . p_{e, f}$ and $q_{e}$ are the transmission failure probability and the probability of at least one packet in the buffer for a safety application, respectively. The state transition diagram of the 1-D Markov chain for the safety applications process is shown in Figure 2 . The nonnull transition probabilities are given by:

$$
\begin{cases}P(k \mid 0)=q_{e} / W_{e}, & 0 \leq k \leq W_{e}-1 \\ P(k \mid k)=p_{e, \text { coll }}, & 1 \leq k \leq W_{e}-1 \\ P(k \mid k+1)=1-p_{e, \text { coll }}, & 0 \leq k \leq W_{e}-2\end{cases}
$$

Here, the non-null transition probabilities describe the unavailability of safety packets transmission in the buffer, hence changing the station into idle $\left(I_{e}\right)$ state after successful transmission is as follows;

$$
\left\{\begin{array}{l}
P\left(I_{e} \mid 0\right)=1-q_{e} \\
P\left(I_{e} \mid I_{e}\right)=1-q_{e} \\
P\left(k \mid I_{e}\right)=q_{e} / W_{e}, \quad 0 \leq k \leq W_{e}-1
\end{array}\right.
$$

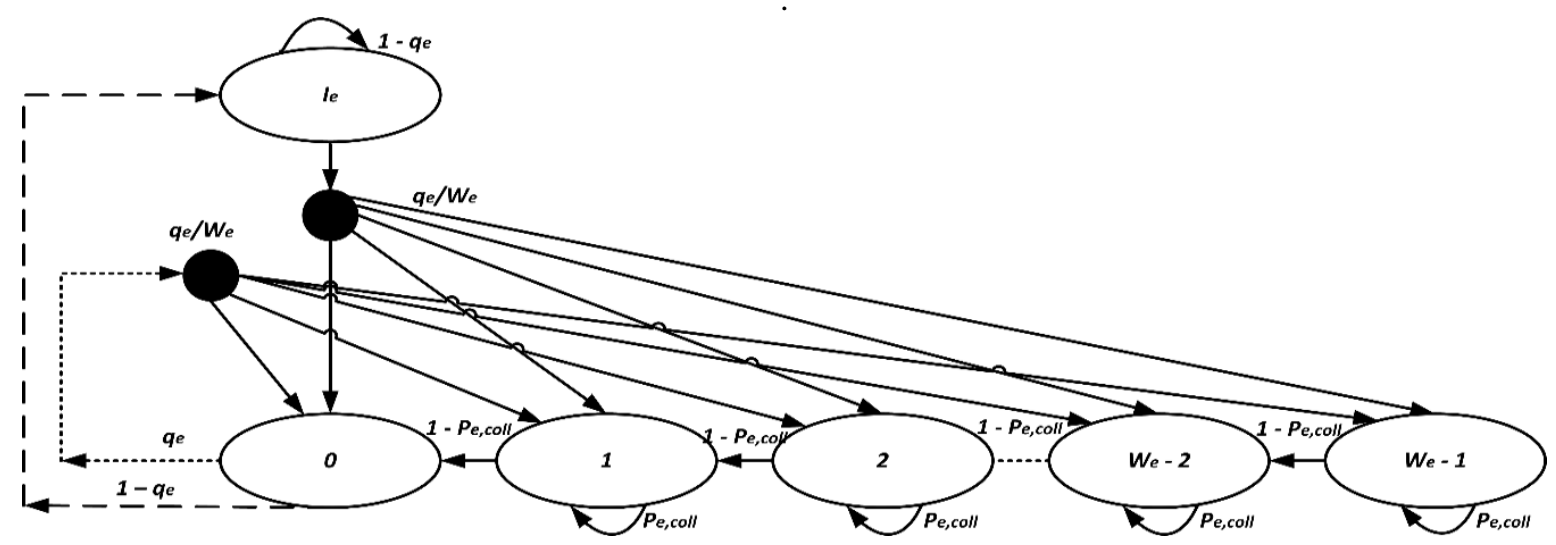

Figure 2. Markov chain model of safety applications

Let $b_{e, k}=\lim _{t \rightarrow \infty} \mathrm{P}\left\{b_{e}(t)=k\right\}$ for $k \in\left(0, W_{e}-1\right)$ be the stationary distribution of the 1-D Markov chain, where $W_{e}$ is the contention window of safety process. From the Markov chain model in Figure 2, we can get:

$$
b_{I_{e}}=\left(1-q_{e}\right) b_{e, 0}+\left(1-q_{e}\right) b_{I_{e}}
$$




$$
\begin{aligned}
& b_{I_{e}}=\frac{1-q_{e}}{q_{e}} b_{e, 0} \\
& b_{e, k}=\frac{W_{e}-k}{W_{e}} \frac{1}{1-p_{e, \text { coll }}} b_{e, 0} \text { for } 1 \leq k \leq W_{e}-1
\end{aligned}
$$

Therefore, by using the normalization condition $1=b_{I_{e}}+\sum_{k=0}^{W_{e}-1} b_{e, k}$ for stationary distribution, we can derive $b_{e, 0}$ as follows:

$$
b_{e, 0}=\frac{2 q_{e}\left(1-p_{e, \text { coll }}\right)}{2\left(1-p_{e, \text { coll }}\right)+q_{e}\left(W_{e}-1\right)}
$$

Let $\tau_{e}$ be the transmission probability of safety applications that a vehicle can transmit in a random chosen time slot. The vehicle can only transmit when the back-off time counter is zero $\left(b_{e, 0}\right)$.

$$
\tau_{e}=b_{e, 0}=\frac{2 q_{e}\left(1-p_{e, c o l l}\right)}{2\left(1-p_{e, c o l l}\right)+q_{e}\left(W_{e}-1\right)}
$$

To analyze $\tau_{s}$, let $s_{s}(t)$ and $b_{s}(t)$ be the random variables representing the back-off stage $(0,1,2, \ldots, m)$ and the value of the back-off timer $\left(0,1,2, \ldots, W_{s, i}-1\right)$ for a given station at time slot $t$, respectively. Typically, the maximum value of the back-off timer relies on the back-off stage; thereby, these random variables are not independent.

$$
W_{s, i}=\left\{\begin{array}{cc}
2^{\mathrm{i}} W_{s, 0}, & i \leq m^{\prime} \\
2^{m^{\prime}} W_{s, 0}, & i>m^{\prime}
\end{array}\right.
$$

where $W_{s, 0}$ is the initial contention window size, $W_{s, 0}=\left(C W_{\min }+1\right)$, and $m^{\prime}$ is the maximum number of trials before the packet is dropped according to $W_{s, m^{\prime}}=2^{m^{\prime}} W_{s, 0}=\left(C W_{s, \max }+1\right)$. The value of $m^{\prime}$ is assumed to be 5. The maximum value of back-off stages is denoted by $m$. The transmission failure probability $p_{s, f}$ is constant and independent in this analysis. So, the two-dimensional $\left(s_{s}(t), b_{s}(t)\right)$ processes are analyzed here with a discrete-time Markov chain at which the channel state changes, as shown in Figure 3 . The state of this process is denoted by $(i, k)$. Thus, the non-null transition probabilities are given by

$$
\begin{cases}P(i, k \mid i, k+1)=1-p_{s, \text { coll }}, & 0 \leq k \leq W_{s, i}-2, \quad 0 \leq i \leq m \\ P(i, k \mid i, k)=p_{s, \text { coll }}, & 1 \leq k \leq W_{s, i}-1, \quad 0 \leq i \leq m \\ P(i, k \mid i-1,0)=p_{s, f} / W_{s, i}, & 0 \leq k \leq W_{s, i}-1, \quad 1 \leq i \leq m \\ P(0, k \mid i, 0)=\left(1-p_{s, f}\right) / W_{s, 0}, & 0 \leq k \leq W_{s, 0}-1, \quad 0 \leq i \leq m \\ P(0, k \mid m, 0)=1 / W_{s, 0}, & 0 \leq k \leq W_{s, 0}-1,\end{cases}
$$

Here, the non-null transition probabilities describe the unavailability of packet transmissions in the buffer which is redirected into idle state $\left(I_{S}\right)$ after a successful transmission.

$$
\left\{\begin{array}{l}
P\left(I_{s} \mid i, 0\right)=\left(1-p_{s, f}\right)\left(1-q_{s}\right), 0 \leq i \leq m-1 \\
P\left(I_{s} \mid m, 0\right)=1-q_{s} \\
P\left(I_{s} \mid I_{s}\right)=1-q_{s} \\
P\left(0, k \mid I_{s}\right)=q_{s} / W_{s, 0}, \quad 0 \leq k \leq W_{s, 0}-1
\end{array}\right.
$$

Let $b_{s, i, k}=\lim _{t \rightarrow \infty} P\left\{s_{s}(t)=i, b_{s}(t)=k\right\}$ be the stationary distribution of the Markov chain, where $i \in(0, m), k \in\left(0, w_{s, i}-1\right)$. First, note that:

$$
\begin{aligned}
& b_{s, i-1,0} \cdot p_{s, f}=b_{s, i, 0} \rightarrow b_{s, i, 0}=p_{s, f}^{i} \cdot b_{s, 0,0} \quad 0<\mathrm{i} \leq \mathrm{m} \\
& b_{s, m, 0}=p_{s, f} b_{s, m-1,0}
\end{aligned}
$$


Due to the chain regularities, for each $\mathrm{k} \in\left(1, W_{s, i}-1\right)$, the stationary distribution of idle and backoff states of service packets are denoted by $b_{I_{S}}$ and $b_{s, i, k}$ and calculated as follows:

$$
b_{s, i, k}=\frac{W_{s, i}-k}{W_{s, i}\left(1-p_{s, \text { coll }}\right)}\left\{\begin{array}{lr}
q_{s}\left(1-p_{s, f}\right) \sum_{i=0}^{m-1} b_{s, i, 0}+q_{s} b_{s, m, 0}+q_{s} b_{I_{s}} & i=0 \\
p_{s, f} \cdot b_{s, i-1,0} & 0<i \leq m
\end{array}\right.
$$

or

$$
\begin{aligned}
& b_{s, i, k}=\frac{W_{s, i}-k}{W_{s, i}} \frac{1}{\left(1-p_{s, \text { coll })}\right.} b_{s, i, 0} \quad \text { for } 0 \leq i \leq m, \quad 1 \leq k \leq W_{s, i}-1 \\
& b_{I_{s}}=\left(1-q_{s}\right)\left(1-p_{s}\right) \sum_{i=0}^{m-1} b_{s, i, 0}+\left(1-q_{s}\right) b_{s, m, 0}+\left(1-q_{s}\right) b_{I_{s}}
\end{aligned}
$$

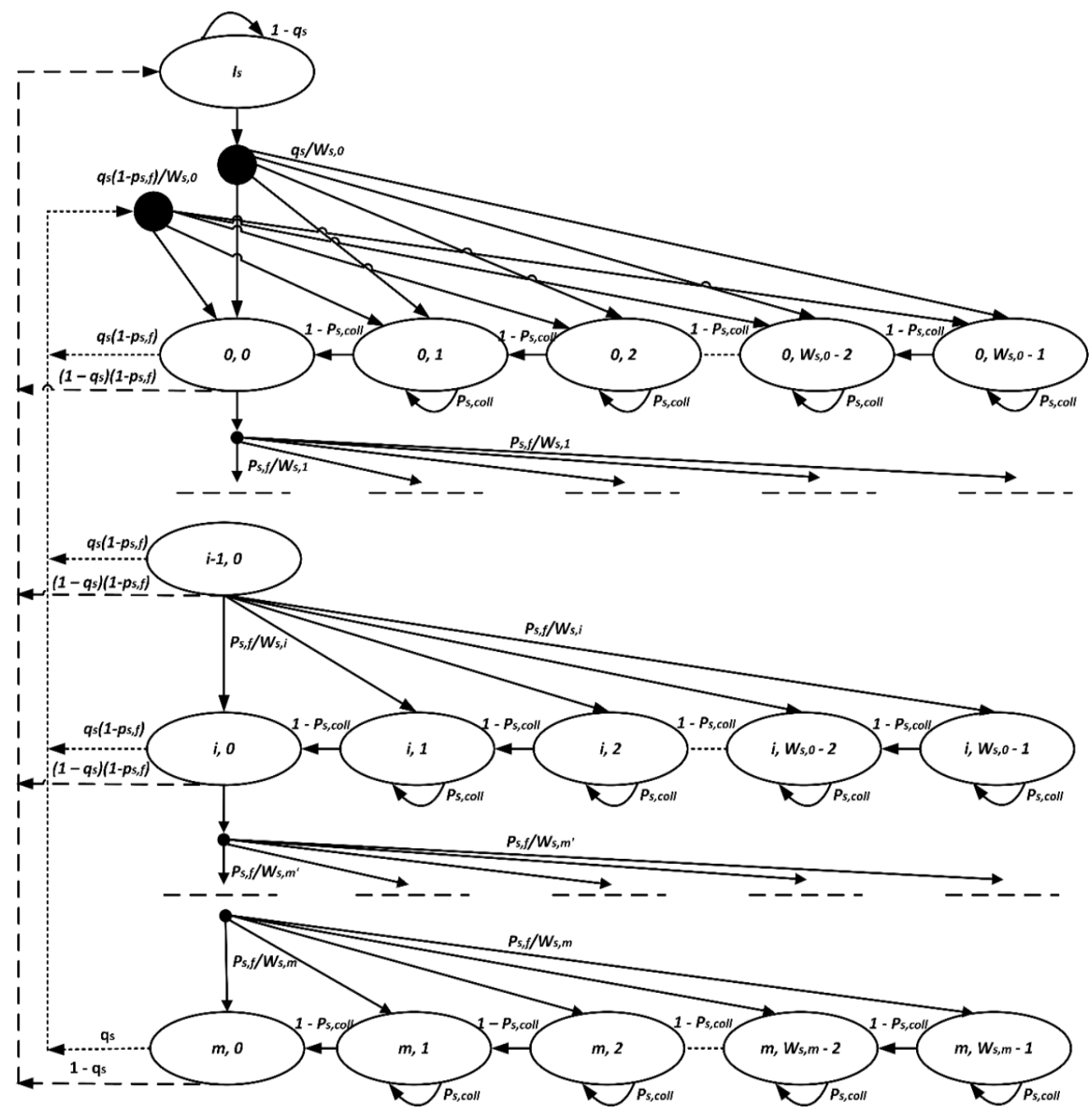

Figure 3. Markov chain model of service applications

By using the normalization condition $1=\sum_{i=0}^{m} \sum_{k=0}^{W_{s, i}-1} b_{s, i, k}+b_{I_{S}}$ for stationary distribution, then $b_{s, 0,0}$ is given by

$$
b_{s, 0,0}= \begin{cases}\frac{2\left(1-p_{s, f}\right)\left(1-p_{s, \text { coll }}\right)\left(1-2 p_{s, f}\right) q_{s}}{£}, & m \leq m^{\prime} \\ \frac{2\left(1-p_{s, f}\right)\left(1-p_{s, \text { coll }}\right)\left(1-2 p_{s, f}\right) q_{s}}{¥}, & m>m^{\prime}\end{cases}
$$


where:

$$
\begin{aligned}
& E=\left(1-2 p_{s, f}\right)\left(1-2 p_{s, \text { coll }}\right)\left(1-p_{s, f}{ }^{m+1}\right) q_{s}+W_{s, 0}\left(1-p_{s, f}\right)\left(1-\left(2 p_{s, f}\right)^{m+1}\right) q_{s}+ \\
& 2\left(1-p_{s, f}\right)\left(1-p_{s, \text { coll }}\right)\left(1-2 p_{s, f}\right)\left(1-q_{s}\right)
\end{aligned}
$$

and:

$$
\begin{aligned}
& ¥=\left(1-2 p_{s, f}\right)\left(1-2 p_{s, \text { coll }}\right)\left(1-p_{s, f}{ }^{m+1}\right) q_{s}+W_{s, 0}\left(1-p_{f}\right)\left(1-\left(2 p_{s, f}\right)^{m^{\prime}+1}\right) q_{s}+ \\
& 2^{m^{\prime}} W_{s, 0} p_{s, f} m^{m^{\prime}+1}\left(1-p_{s, f}{ }^{m-m^{\prime}}\right)\left(1-2 p_{s, f}\right) q_{s}+2\left(1-p_{s, f}\right)\left(1-p_{s, \text { coll }}\right)\left(1-2 p_{s, f}\right) q_{s}
\end{aligned}
$$

Let $\tau_{s}$ be the transmission probability of service applications that a vehicle can transmit a service packet in a random chosen time slot. The vehicle can only transmit the packet when the back-off time counter is zero $\left(b_{s, \mathrm{i}, 0}\right)$ regardless of the back-off stage.

$$
\tau_{s}=\sum_{i=0}^{m} b_{s, i, 0}=b_{s, 0,0} \frac{1-p_{s, f} m+1}{1-p_{s, f}}
$$

\subsection{Failure $\mathbf{p}_{\mathbf{f}}$ and collision $\mathbf{p}_{\text {coll }}$ probabilities}

The transmission failure probabilities $p_{e, f}$ and $p_{s, f}$ of safety and service packets are respectively derived as follows:

$$
\left\{\begin{array}{l}
p_{e, f}=1-\left(1-p_{e, \text { coll }}\right)\left(1-p_{e, e r r}\right) \\
p_{s, f}=1-\left(1-p_{s, \text { coll }}\right)\left(1-p_{s, e r r}\right)
\end{array}\right.
$$

where $p_{e, e r r}$ and $p_{s, e r r}$ denote the probability of frame error for safety and service packets, respectively.

$$
\left\{\begin{array}{l}
p_{e, \text { err }}=1-\left(1-p_{e, s a f_{\_} \text {err }}\right) \\
p_{s, \text { err }}=1-\left(1-p_{s, \text { wsa_err }}\right)\left(1-p_{s, r f s_{\_} e r r}\right)\left(1-p_{s, a c k \_e r r}\right)
\end{array}\right.
$$

where $p_{e, s a f \_e r r}, p_{s, \text { wsa_err }}, p_{s, r f s_{\text {_err }}}$, and $p_{s, \text { ack_err }}$ denote the Frame Error Rate (FERs) for safety and WSA/RFS/ACK frames error, respectively. The probability of these errors can be computed from bit error probability (i.e. BER) $p_{B E R}$ as follows [9]:

$$
\left\{\begin{array}{l}
p_{e, s a f \_e r r}=1-\left(1-p_{B E R}\right)^{L_{s a f}} \\
p_{s, w s a \_e r r}=1-\left(1-p_{B E R}\right)^{L_{w s a}} \\
p_{s, r e q \_e r r}=1-\left(1-p_{B E R}\right)^{L_{r f s}} \\
p_{s, a c k \_e r r}=1-\left(1-p_{B E R}\right)^{L_{a c k}}
\end{array}\right.
$$

where $L_{s a f}, L_{w s a}$, and $L_{a c k}$ represents the size of safety and WSA/RFS/ACK frames respectively. The probabilities of collision for safety $p_{e, \text { coll }}$ and service $p_{s, \text { coll }}$ packets are respectively defined as follows:

$$
\left\{\begin{array}{l}
p_{e, \text { coll }}=1-\left(1-\tau_{e}\right)^{n-1}\left(1-\tau_{s}\right)^{n} \\
p_{s, \text { coll }}=1-\left(1-\tau_{s}\right)^{n-1}\left(1-\tau_{e}\right)^{n}
\end{array}\right.
$$

then,

$$
\left\{\begin{array}{l}
p_{e, f}=1-\left(1-\tau_{e}\right)^{n-1}\left(1-\tau_{s}\right)^{n}\left(1-p_{e, e r r}\right) \\
p_{s, f}=1-\left(1-\tau_{s}\right)^{n-1}\left(1-\tau_{e}\right)^{n}\left(1-p_{s, e r r}\right)
\end{array}\right.
$$

From (7), (18), and (23), we can solve the two unknown variables, $\tau_{e}, \tau_{s}, p_{e, f}$ and $p_{s, f}$ by using numerical techniques in order to calculate the transmission and failure probabilities for safety and service applications respectively. 


\subsection{Time analysis for safety and WSA transmission}

In every time slot during the contention-based MAC scheme $(\mathrm{CCH})$, the state of the channel could be idle $p_{e s, i}$, successful transmission, collision transmission or failure transmission due to the frame error. Thus, the probabilities of channel states are expressed by (24).

$$
\left\{\begin{array}{l}
p_{e s, i}=\left(1-\tau_{e}\right)^{n}\left(1-\tau_{s}\right)^{n} \\
p_{e, s u c}=n \tau_{e}\left(1-\tau_{e}\right)^{n-1}\left(1-\tau_{s}\right)^{n}\left(1-p_{e, s a f_{\_} e r r}\right) \\
p_{e, S A F_{-} E R R}=n \tau_{e}\left(1-\tau_{e}\right)^{n-1}\left(1-\tau_{s}\right)^{n} p_{e, s a f_{-} \text {err }} \\
p_{s, s u c}=n \tau_{s}\left(1-\tau_{s}\right)^{n-1}\left(1-\tau_{e}\right)^{n}\left(1-p_{s, w s a_{-} e r r}\right)\left(1-p_{s, r f s_{-} e r r}\right)\left(1-p_{s, a c k \_e r r}\right) \\
p_{s, W S A_{-} E R R}=n \tau_{s}\left(1-\tau_{s}\right)^{n-1}\left(1-\tau_{e}\right)^{n} p_{s, w s a_{-} e r r} \\
p_{s, R F S_{-} E R R}=n \tau_{s}\left(1-\tau_{s}\right)^{n-1}\left(1-\tau_{e}\right)^{n}\left(1-p_{s, w s a_{-} e r r}\right) p_{s, r f s_{-} e r r} \\
p_{s, A C K_{-} E R R}=n \tau_{s}\left(1-\tau_{s}\right)^{n-1}\left(1-\tau_{e}\right)^{n}\left(1-p_{s, w s a \_e r r}\right)\left(1-p_{s, r f s_{\text {err }}}\right) p_{s, a c k \_e r r} \\
p_{e s, b}=1-p_{e s, i}=1-\left(1-\tau_{s}\right)^{n}\left(1-\tau_{e}\right)^{n} \\
p_{e, c}=\left(1-\tau_{s}\right)^{n}\left(1-\left(1-\tau_{e}\right)^{n}-n \tau_{e}\left(1-\tau_{e}\right)^{n-1}\right) \\
p_{s, c}=\left(1-\tau_{e}\right)^{n}\left(1-\left(1-\tau_{s}\right)^{n}-n \tau_{s}\left(1-\tau_{s}\right)^{n-1}\right) \\
p_{e s, c}=p_{e s, b}-p_{e, s u c}-p_{s, s u c}-p_{e, c}-p_{s, c}
\end{array}\right.
$$

where $p_{e, s u c}$ and $p_{s, s u c}$ represent the successful transmission probabilities of safety and service packets, respectively. $p_{e, S A F_{-} E R R}, p_{S, W S A_{E R R}}, \quad p_{S, R F S_{-} E R R}$ and $p_{S, A C K_{-} E R R}$ denote the unsuccessful transmission probabilities of safety and WSA/RFS/ACK packets due to the frames error. The busy channel probability is denoted by $p_{e s, b}$. The transmission probability of a safety packet with collision caused by safety packets only is denoted by $p_{e, c}$. The transmission probability of a service packet with collision caused by service packets only is denoted by $p_{s, c}$. The transmission probability of a packet with collision caused by safety and service packets.

Now, let $T_{e s, i}$ denotes the idle slot duration. $T_{e, s u c}$ and $T_{s, s u c}$ represent the successful transmission time of safety and service packets, respectively. $T_{e, c}$ and $T_{S, c}$ denote the transmission time of safety and service packets with collision, respectively. $T_{e, S A F_{-} E R R}, T_{S, W S A_{-} E R R}, T_{S, R F S_{-} E R R}$, and $T_{S, A C K_{-} E R R}$ denote the unsuccessful transmission times of safety and WAS/RFS/ACK packets due to frames error. $T_{S a f}, T_{W S A}, T_{R F S}$, and $T_{A C K}$ are the time duration to transmit safety, and WSA/RFS/ACK packets respectively. $T_{S I F S}, T_{D I F S}$, and $T_{E I F S}$ are the time duration of SIFS, DIFS and EIFS respectively. $\delta$ is the propagation delay. $\sigma$ is the idle time slot. Thus, the transmission time with adoption broadcast and unicast mechanisms is calculated by

$$
\left\{\begin{array}{l}
T_{e s, i}=\text { aSlotTime }=\sigma \\
T_{e, s u c}=T_{e, c}=T_{e, S A F_{-} E R R}=T_{s a f}+\delta+T_{D I F S} \\
T_{S, S u c}=T_{w s a}+T_{r f s_{S}}+T_{a c k}+3 \delta+2 T_{S I F S}+T_{D I F S} \\
T_{s, c}=T_{w s a}+\delta+T_{E I F S} \\
T_{S, W S A_{-} E R R}=T_{w s a}+\delta+T_{E I F S} \\
T_{S, R F S_{-} E R R}=T_{w s a}+T_{S I F S}+T_{r f s}+2 \delta+T_{E I F S} \\
T_{S, A C K_{-} E R R}=T_{S, S u c}
\end{array}\right.
$$

However, assuming that the service data packet size is constant, then the time slot duration to transmit a service data packet over the $\mathrm{SCH}$ based on the contention-free MAC scheme is expressed by

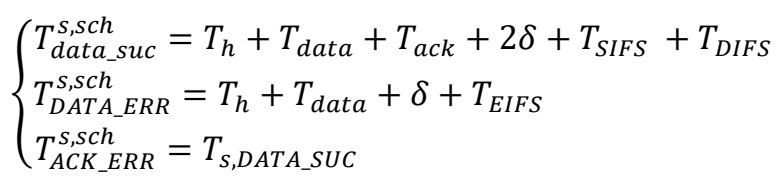

where $T_{\text {data_suc }}^{\text {S,sch }}$ denotes the successful transmission time of service data packet over SCH. $T_{D A T A \_E R R}^{S, S C h}$ is the unsuccessful transmission time of a service data packet over $\mathrm{SCH}$ due to frame error. $T_{h}=P H Y_{h d r}+$ $M A C_{h d r}$ is the packet header. $T_{d a t a}=L_{p l d} / R$, where $L_{p l d}$ represents the payload of the service data packet, and $\mathrm{R}$ is the transmission data rate. $T_{s a f}, T_{w s a}, T_{r f s}$ and $T_{a c k}$ are PHY-layer dependent, and the frame transmissions in the unit of Orthogonal Frequency Division Multiplexing (OFDM) symbols are given by (27) [19]: 


$$
\left\{\begin{array}{c}
T_{\text {saf }}=T_{\text {symbol }}\left[\frac{L_{\text {ser }}+L_{\text {tail }}+L_{\text {saf }}}{N_{\text {Bps }}}\right\rceil \\
T_{\text {wsa }}=T_{\text {symbol }}\left[\frac{L_{\text {ser }}+L_{\text {tail }}+L_{w s a}}{N_{\text {Bps }}}\right] \\
T_{r f s}=T_{\text {symbol }}\left[\frac{L_{\text {ser }}+L_{\text {tail }}+L_{r f s}}{N_{\text {Bps }}}\right\rceil \\
T_{\text {data }}=T_{\text {symbol }}\left[\frac{L_{\text {ser }}+L_{\text {tail }}+L_{\text {data }}}{N_{\text {Bps }}}\right] \\
T_{\text {ack }}=T_{\text {symbol }}\left[\frac{L_{\text {ser }}+L_{\text {tail }}+L_{\text {ack }}}{N_{\text {Bps }}}\right]
\end{array}\right.
$$

where $T_{\text {symbol }}, L_{\text {ser }}, L_{\text {tail }}$, and $N_{B p S}$ denote the duration of a transmission symbol, OFDM PHY layer service field size, OFDM PHY layer tail fields size, and the number of encoded bites per one symbol. In order to figure out the system throughput and delay, the duration of the logical time slots $T_{\text {slot }}$ per state in the channel is required and given by:

$$
\begin{aligned}
& T_{\text {slot }}=p_{e s, i} T_{e s, i}+p_{e, s u c} T_{e, s u c}+p_{s, s u c} T_{S, S u c}+p_{e, c} T_{e, c}+p_{s, c} T_{s, c}+p_{e, S A F_{-} E R R} T_{e, S A F_{-} E R R}+ \\
& p_{s, W S A_{-} E R R} T_{S, W S A_{-} E R R}+p_{s, R F S_{-} E R R} T_{S, R F S_{-} E R R}+p_{s, A C K_{-} E R R} T_{s, A C K_{-} E R R}+p_{e s, c} \max \left(T_{e, c}, T_{s, c}\right)
\end{aligned}
$$

In this model, the Poisson distribution model is assumed, in which the inter arrival time is exponentially distributed. Then, from the average duration of the logical time slot $T_{\text {slot }}$, the load equation of queue probability, $q_{e}$ and $q_{s}$ for safety and service applications is given respectively by [10]:

$$
\left\{\begin{array}{l}
q_{e}=1-e^{-2 \lambda_{e} T_{s l o t}} \\
q_{s}=1-e^{-2 \lambda_{s} T_{s l o t}}
\end{array}\right.
$$

The packet delivery ratio (PDR) of the safety application is derived as the probability of having a successful transmission during a given time slot over the average number of vehicles transmitting packets in a generic time slot;

$$
P D R=\frac{p_{e, s u c}}{n_{e} \tau_{e}}=\left(1-\tau_{e}\right)^{n-1}\left(1-\tau_{s}\right)^{n}
$$

The average time slot of a safety packet to execute the back-off is given by $\frac{\left(w_{e}-1\right)}{2}$. Thereby, the average total service time $E\left[X_{e}\right]$ of a safety packet, which experiences the average back-off duration, can be estimated by

$$
\mu_{e}=E\left[X_{e}\right]=\frac{\left(w_{e}-1\right)}{2} * T_{\text {slot }}
$$

In this model, each vehicle is modelled as an $\mathrm{M} / \mathrm{M} / 1$ queue with an infinitive buffer size, service rate $\mu_{e}$, and the packet arrival rate $2 \lambda_{e}$. In 1609.4 standard, the CCHI and SCHI have the same duration (50 $\mathrm{ms}$ for each interval), thereby, the average arrived packets are equal. However, safety packets which are generated during SCHI have to delay by $T_{c c h i}$. Therefore, the average delay of safety packets $E\left[D_{e}\right]$ including queuing and transmission delays is expressed by

$$
E\left[D_{e}\right]=\frac{2 \mu_{e}}{\left(1-2 \lambda_{e} \mu_{e}\right)}+T_{e, s u c}+\frac{T_{c c h i}}{2}
$$

However, the retry limit is considered in the model analysis of the WSAs packets in order to meet the IEEE 802.11 p specifications. Thus, the maximum back-off stage for the WSA packet to be transmitted in this model is denoted by $\mathrm{m}$. If the WSA packet faces $\mathrm{m}$ collisions in the previous stages, and therefore this packet will be dropped if it experiences another collision. Then, the WSA packet drop probability $P_{S, d r o p}$ expressed by

$$
P_{s, \text { drop }}=p_{s, f}^{m+1}
$$

For service applications, when the vehicles successfully exchange the WSA packets over CCH, they will tune to the selected SCH during SCHI to transfer service data. The maximum time that vehicles use to 
exchange service data is one SCHI $(50 \mathrm{~ms})$. In this model, the SCHI is divided into $Q$ transmission slots. Initially, vehicles have to exchange WSA packets over $\mathrm{CCH}$ during $\mathrm{CCHI}$ to choose suitable transmission slot for their service data transmission over SCHs. Thus, the average number of WSA packets $N_{s, s u c}$ successfully transmitted over the $\mathrm{CCH}$ during the $\mathrm{CCHI}$ is calculated by:

$$
N_{s, s u c}=\frac{T_{c c h i}}{T_{s l o t}} \times p_{s, s u c}
$$

According to the 1609.4 standard, the number of service channel is six, thus, the maximum transmission slots can be utilized is 6Q. Finally, the aggregate throughput of the service packets $S_{S}$, is evaluated by considering the number of selected transmission slots, and thus, it can be estimated by [17],

$$
S_{s}=\min \left[N_{s, \text { suc }}, 6 Q\right]
$$

\section{MODEL VALIDATION}

We use MATLAB to carry out the numerical results, while the extensive simulations are conducted to validate the proposed analytical model using NS-2.34. The simulation scenario includes 100 vehicles with a GPS and a single-radio WAVE communication device. The speed of vehicles is $60 \mathrm{~km} / \mathrm{h}$. The value of bit error rate $p_{B E R}$ for the channel condition is assumed to be $10^{-5}$, which is one of the most affected and sensitive values for the channel BER in a comparatively noisy, channel fading and unreliable wireless environment [20-21]. The typical parameters values for both analytical model and simulations are summarized in Table 1 as obtained from [17]. The safety packet arrival rate $\lambda_{e}$ is varied up to 100 packets/second (pps), while the service packet arrival rate $\lambda_{s}$ and the number of vehicles $n$ are fixed, $\lambda_{s}=50 p p s$ and $n=30$ vehicle. Performance metrics such as average delay and PDR of safety packets, as well as the network throughput of service packets are investigated in this section.

Table 1. Parameters values

\begin{tabular}{cccc}
\hline Parameters & value & Parameters & value \\
\hline Number of CCH & 1 & $\delta$ & $1 \mu \mathrm{s}$ \\
Number of SCHs & 6 & $L_{\text {ser }}$ & 16 bits \\
Transmission data rate for & $6 \mathrm{Mbps}$ & $L_{\text {tail }}$ & $6 \mathrm{bits}$ \\
each channel & $272 \mathrm{bits}$ & $p_{B E R}$ & $10^{-5}$ \\
MAC header & $128 \mathrm{bits}$ & $T_{\text {symbol }}(\mu \mathrm{s})$ & 4 \\
PHY header & $8000 \mathrm{bits}$ & $N_{B p S}$ & 24 \\
Service packet size & $800 \mathrm{bits}$ & $W_{e}$ & 8 \\
Safety packet size & $800 \mathrm{bits}$ & $W_{s, 0}$ & 16 \\
WSA & $160 \mathrm{bits}$ & Short retry limit & 5 \\
RFS & $112 \mathrm{bits}$ & Long retry limit & 7 \\
ACK & $16 \mu \mathrm{s}$ & Number of transmission slots $Q$ & 6 \\
SIFS & $9 \mu \mathrm{s}$ & Frequency & $5.9 \mathrm{GHz}$ \\
Time slot $\sigma$ & $34 \mu \mathrm{s}$ & $\lambda_{e}$ & $10-100 \mathrm{pps}$ \\
DIFS & & &
\end{tabular}

Figure 4 displays the performance of the IEEE 1609.4 MAC in VANETs under various safety packet arrival rate $\lambda_{e}$. In fact, the value of collision probability increases with increasing the packet arrival rate or the number of vehicles in the network. The reason for this can be attributed to the fact that in the heavy packet arrival rate (traffic load), more broadcasting packets are exchanged, leading to a higher collision probability. In addition, due to the lack of ACK and exponential back-off mechanisms in safety packets broadcast, vehicles with broadcast mode attempts to transmit a packet after the last packet being broadcasted constantly, which results in higher collision probability. According to these facts, we observe the PDR of safety packets in Figure 4(b) and the throughput of service packets in Figure 4(d) decrease with increasing the packet arrival rat in the network. We also notice in Figure 4(c) that the WSA packets drop probability significantly increases with increasing safety packet arrival rate. Figure 4(a) shows that the average delay of safety packets increases linearly with increasing packet arrival rate. This observation explains that the average service time $E\left[X_{e}\right]=\mu_{e}$ is directly proportional to the packet arrival rate. Explicitly, as the packet arrival rate increases, the packets queue and service time increase as well and this certainly lead to longer delay for packets to be transmitted. In addition, with more safety packets being broadcasted, the back-off time occurs more frequently and the overall back-off time increases for each vehicle in the network due to more frequent freezing of the back-off timer. 

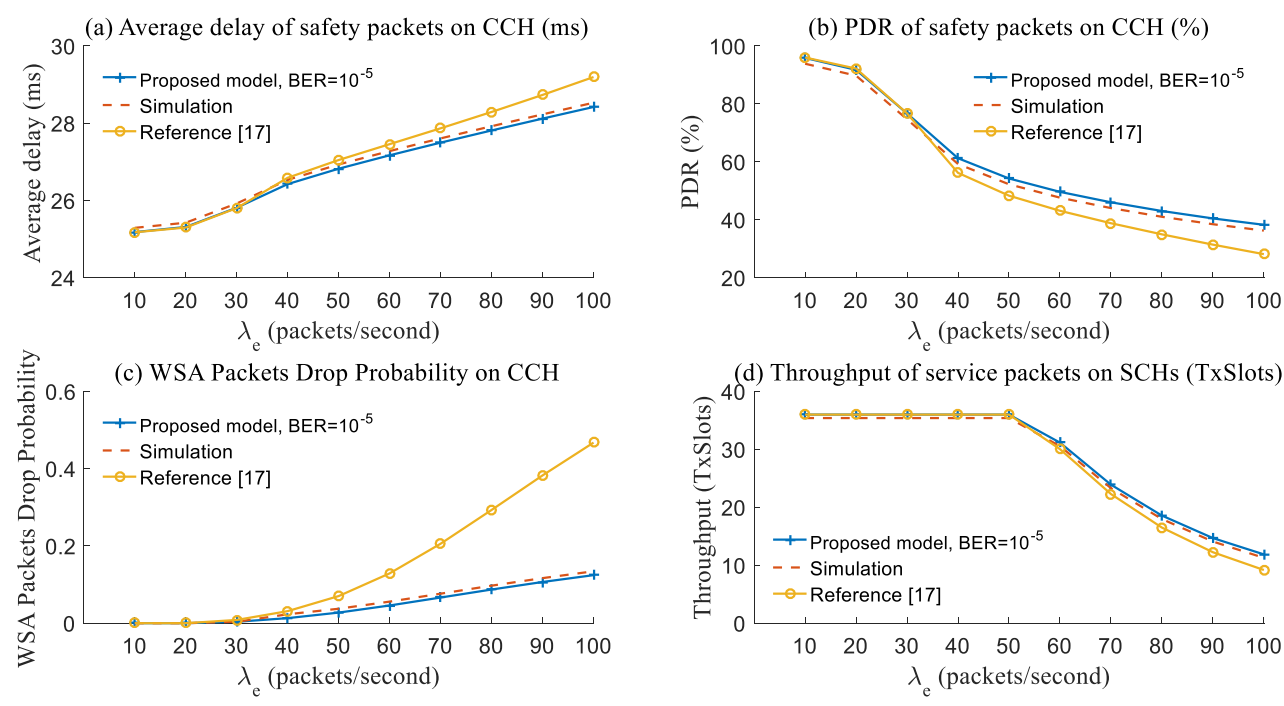

Figure 4. Performance analysis of the IEEE 1609.4, $\lambda_{s}=50$ pps and $n=30$ vehicle

Figure 4 clearly shows that the results of the proposed model outperform the results of the existing model, although the BER is considered in the proposed model. This is due to the freezing of the back-off timer mechanism that is taken into consideration in the proposed model. This mechanism always keeps the vehicles aware of the channel status to apply the freezing of the back-off timer when there is a collision in the channel to reduce the collision probability, especially when the packet arrival rate increases in the network. In addition, the simulation results in Figure 4 are close to the analytical results, which validate the accuracy of the proposed model.

\section{CONCLUSION}

This paper has proposed analytical models based on 1-D and 2-D Markov chain for safety and service applications, respectively, to evaluate the performance of the IEEE 1609.4 MAC in in the presence of error prone channels. The result displays the effect of the packet arrival rate on the network performance. The network performance degrades with increasing the safety packet arrival rate. The study also shows that the results of the proposed model outperform the results of the existing model in terms of average delay and PDR of safety packets over $\mathrm{CCH}$, and network throughput of service packets over SCHs.

\section{REFERENCES}

[1] "IEEE Standard for Wireless Access in Vehicular Environments (WAVE)-Multi-channel Operation," IEEE Std 1609.4-2010, (Revision of IEEE Std 1609.4-2006), 2011.

[2] A. A. Almohammedi, et al., "An Adaptive Multi-Channel Assignment and Coordination Scheme for IEEE 802.11 P/1609.4 in Vehicular Ad-Hoc Networks," IEEE Access, vol. 6, pp. 2781-2802, 2018.

[3] A. A. Almohammedi, et al., "An accurate performance analysis of hybrid efficient and reliable MAC protocol in VANET under non-saturated conditions," International Journal of Electrical and Computer Engineering, vol. 7, pp. 999, 2017.

[4] W. A. Jabbar, et al., "MEQSA-OLSRv2: A Multicriteria-Based Hybrid Multipath Protocol for Energy-Efficient and QoS-Aware Data Routing in MANET-WSN Convergence Scenarios of IoT," IEEE Access, vol. 6, pp. 7654676572, 2018.

[5] W. A. Jabbar, et al., "Design and Implementation of IoT-Based Automation System for Smart Home," in 2018 International Symposium on Networks, Computers and Communications (ISNCC), pp. 1-6, 2018.

[6] G. Bianchi, "Performance analysis of the IEEE 802.11 distributed coordination function," IEEE Journal on selected areas in communications, vol. 18, pp. 535-547, 2000.

[7] H. Wu, et al., "Performance of reliable transport protocol over IEEE 802.11 wireless LAN: analysis and enhancement," in INFOCOM 2002. Twenty-First Annual Joint Conference of the IEEE Computer and Communications Societies. Proceedings. IEEE, pp. 599-607, 2002.

[8] F. Daneshgaran, et al., "Saturation throughput analysis of IEEE 802.11 in the presence of non ideal transmission channel and capture effects," IEEE transactions on Communications, vol. 56, 2008. 
[9] K. Szczypiorski and J. Lubacz, "Saturation throughput analysis of IEEE 802.11 g (ERP-OFDM) networks," Telecommunication Systems, vol. 38, pp. 45-52, 2008.

[10] D. Malone, et al., "Modeling the 802.11 distributed coordination function in nonsaturated heterogeneous conditions," IEEE/ACM Transactions on networking, vol. 15, pp. 159-172, 2007.

[11] N. Gupta and C. S. Rai, "Performance evaluation of IEEE 802.11 DCF in single Hop Ad Hoc networks," Wireless personal communications, vol. 79, pp. 2171-2193, 2014.

[12] R. Woo, et al., "Performance analysis for priority-based broadcast in vehicular networks," International Journal of Distributed Sensor Networks, vol. 9, pp. 734637, 2013.

[13] K. A. Hafeez, et al., "Impact of mobility on vanets' safety applications," in Global Telecommunications Conference (GLOBECOM 2010), 2010 IEEE, pp. 1-5, 2010.

[14] A. J. Ghandour, et al., "Dissemination of safety messages in IEEE 802.11 p/WAVE vehicular network: Analytical study and protocol enhancements," Pervasive and mobile computing, vol. 11, pp. 3-18, 2014.

[15] P. Rathee, et al., "Performance Analysis of IEEE $802.11 \mathrm{p}$ in the Presence of Hidden Terminals," Wireless Personal Communications, vol. 89, pp. 61-78, 2016.

[16] A. A. A. Almohammedi, et al., "A Comprehensive Performance Analysis of IEEE 802.11 p based MAC for Vehicular Communications Under Non-saturated Conditions," Journal of ICT Research and Applications, vol. 11, pp. 91-112, 2017.

[17] D. N. M. Dang, et al., "Analytical Study of the IEEE 1609.4 MAC in Vehicular Ad Hoc Networks," in International Conference on Advanced Engineering Theory and Applications, pp. 145-154, 2017.

[18] C. Song, et al., "APDM: An adaptive multi-priority distributed multichannel MAC protocol for vehicular ad hoc networks in unsaturated conditions," Computer Communications, vol. 104, pp. 119-133, 2017.

[19] "Part 11: Wireless LAN Medium Access Control (MAC) and Physical Layer (PHY) Specifications: High-speed Physical Layer in the 5 GHz Band," IEEE Std 802.11a-1999 (1999). (8802-11:1999/Amd 1:2000(E)).

[20] Q. Ni, et al., "Saturation throughput analysis of error-prone 802.11 wireless networks," Wireless Communications and Mobile Computing, vol. 5, pp. 945-956, 2005.

[21] D. J. Deng, et al., "Saturation throughput analysis of multi-rate IEEE 802.11 wireless networks," Wireless Communications and Mobile Computing, vol. 9, pp. 1102-1112, 2009. 